\title{
Research about the Evaporative Cooling Sleeve of 3.6 MW Wind Generator Stator
}

\author{
Shunzhou Yu *, Jie Yang *, Jiayi Yuan *, and Xindong Tian *
}

\begin{abstract}
The evaporative cooling technology used in Wind generator stator has unique advantages. Combined with the structure of motor stator and operating conditions, this report based on the research project for the evaporative cooling sleeve of the $3.6 \mathrm{MW}$ wind generator, introduces the material requirements and structural characteristics of the sleeve, simulates on the stress, displacement and stability by finite analysis method, and tests the products experimentally. The research results show that the epoxy resin-glass materials have a higher strength and better insulation properties, but the evaporative cooling of the wind generator stator sleeve, because of its thin-walled, and the external pressure, so it's the less rigid. Should make full use of the motor stator core structure, increase its stiffness and improve the stability of the epoxy resin-glass sleeve, which for thin-walled the epoxy resinglass sleeve on the successful application of wind turbines has played an important role.
\end{abstract}

Keywords: Evaporative cooling, Wind generator, Epoxy resin-glass sleeve, Stability

\section{Introduction}

Generator stator with immersion evaporative cooling technology, the stator cavity is made completely sealed, the liquid medium which is filled with low boiling point, high insulation, not combustion, non-toxic, stable chemical properties [1]. When the motor is running, the stator cavity coil winding, iron core and within all structural components, can be fully cooling. The motor temperature level is low, and its uniform distribution. Compared with other cooling methods, the cooling efficiency is higher, the electric load density greater [2]. Therefore, the equivalent capacity of the motor is smaller size, lighter weight, higher material utilization rate, is a kind of advanced cooling method.

When the wind generator working on the tower tens or even hundreds of meters from the ground, which has a higher requirement on transportation, installation, operation and maintenance, the evaporative cooling method has the unique advantages[3]. The key member of this cooling method is the sleeve in the stator cavity, which is installed in the air gap between the motor stator and rotor. In order not to affect the motor magnetic circuit design, the sleeve cannot be used magnetic materials. At the same time, to avoid a large amount of eddy current loss, it is necessary to adopt a non-magnetic insulating material [3]. In addition, the sleeve wall if it is too thick, the air gap between rotor

Institute of Electrical Engineering, Chinese Academy of Sciences,

Beijing, China. (kezhi@mail.iee.ac.cn)

Received 15 August 2013; Accepted 10 October 2013 and stator will increase, so, in order to meet the need of electromagnetic field, the permanent magnet of rotor will increase, which will lead to increase the economic cost of the motor. Because the entire stator cavity is sealed to withstand the pressure, so the wall thickness of the sleeve cannot be too thin, otherwise its mechanical strength is not up to the requirements. Then, the material must be compatible with the evaporative cooling medium. After a lot of experiment research, this report adopts the epoxy resin-glass material which has high strength.

\section{Structural Features}

The epoxy resin-glass material of the sleeve is anisotropic, and its basic properties are as follows:

$\mathrm{Ex}=51 \mathrm{GPa}, \mathrm{Ex}=\mathrm{Ez}=10.7 \mathrm{GPa}, \mathrm{Vxy}=\mathrm{Vxz}=0.38, \mathrm{Vyz}$ $=0.31$, Gxy $=\mathrm{Gxz}=5.2 \mathrm{GPa}, \mathrm{Gyz}=2.6 \mathrm{GPa}$. Where, $\mathrm{X}$ direction of the fiber orientation.

The external pressure on the sleeve is about $0.06 \mathrm{MPa}$ in the normal operating conditions. In this paper, Simulation design pressure is $0.2 \mathrm{MPa}$, in the actual test, the maximum pressure is $0.16 \mathrm{MPa}$, to ensure the normal operation of the motor with a sufficient safety factor.

According to the electromagnetic calculation and overall design of the $3.6 \mathrm{MW}$ wind generator, the diameter of sleeve is $1.3 \mathrm{~m}$, the length and thickness of the thin-walled section is $1.5 \mathrm{~m}$ and $4.5 \mathrm{~mm}$. In order to be sealed easily, the sealing surface of the sleeve ends is thicker and the middle section 
is thinner, so the thickness of the sleeve is gradient and elimination of stress is centralized. The structure of the sleeve shown in Fig. 1, and Fig. 2 is a photograph of the product sleeve.

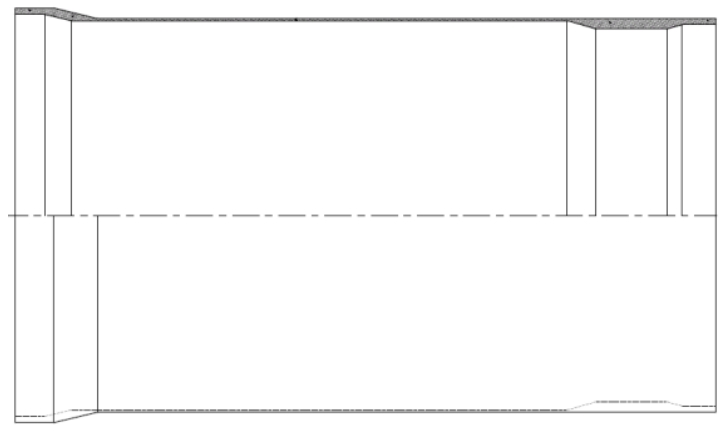

Fig. 1. Sleeve structure

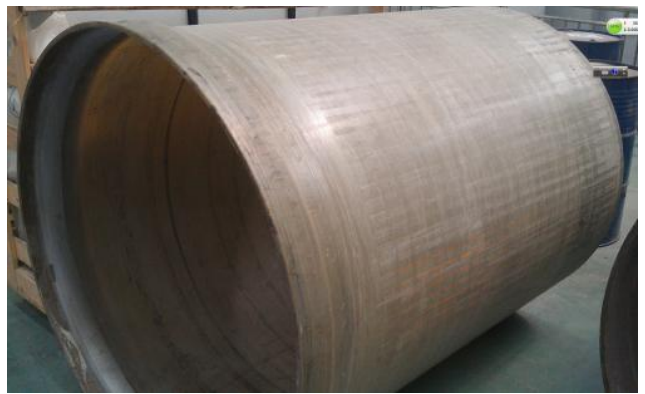

Fig. 2. Sleeve products

\section{Simulation and Calculation}

Based on the physical dimensions of the design, we establish a numerical model and analyze the model by finite element method of hexahedral meshing, finite element model of the sleeve shown in Fig. 3. When the sleeve is subjected to external pressure of $0.2 \mathrm{MPa}$ loads, a core and non-core two cases for stress, displacement, and stability in the simulation, sleeve stress nephogram, radial displacement nephogram and first order buckling mode chart, as shown in Figs. 4 to 9.

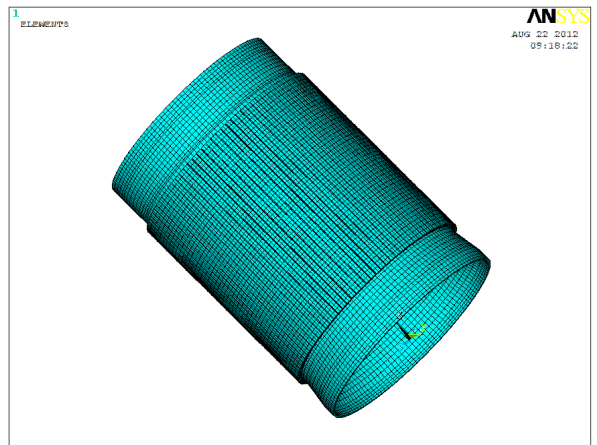

Fig. 3. Finite element model of the sleeve

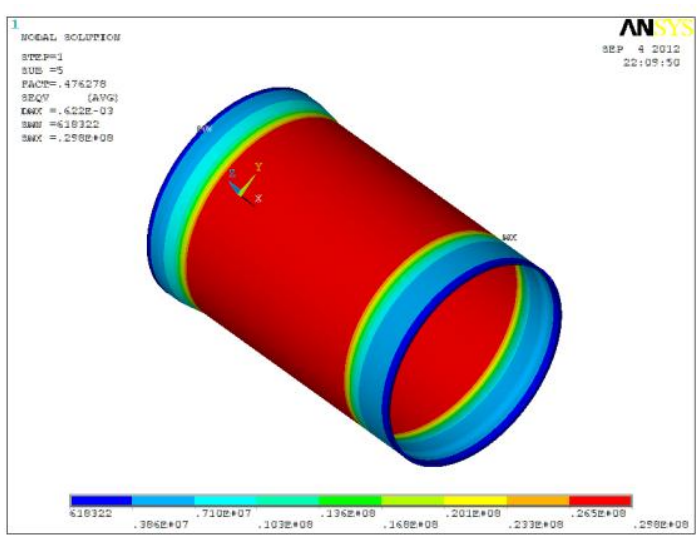

Fig. 4. Sleeve stress nephogram (Coreless Maximum stress $29.8 \mathrm{MPa}$ )

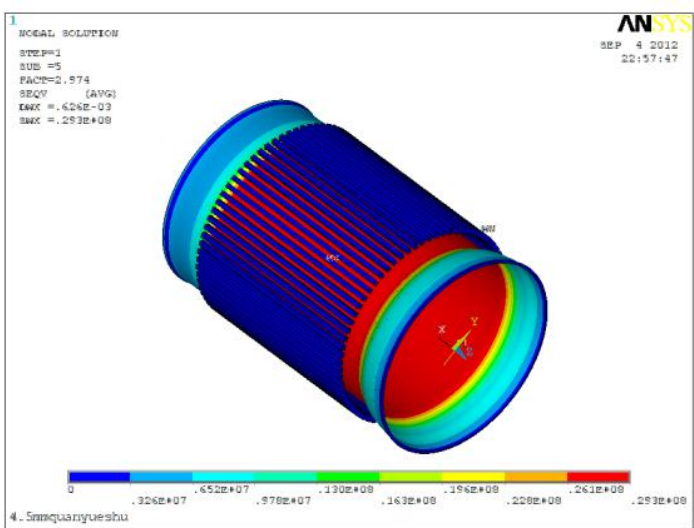

Fig. 5. Sleeve stress nephogram (Core Maximum stress 29.3MPa)

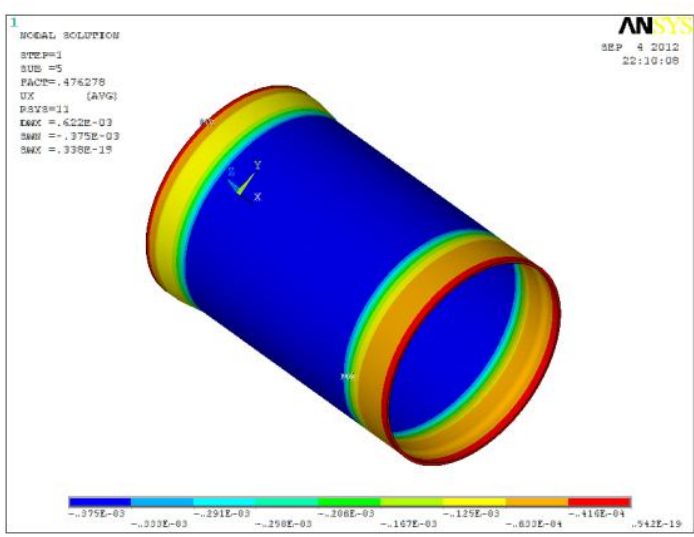

Fig. 6. The radial displacement nephogram (Coreless) (The maximum displacement $-0.375 \mathrm{~mm}$ )

The results show that thin-walled part of the central sleeve is weak link, and is symmetrically distributed. In the core and non core condition, the generated stress is basically the same; its maximum stress (30MPa or so) is far less than the allowable stress of epoxy resin-glass material. The radial displacement has little difference, the maximum radial displacement is $-0.4 \mathrm{~mm}$ ("-" said displacement 
direction toward the smaller diameter direction), and can also meet the using requirements.

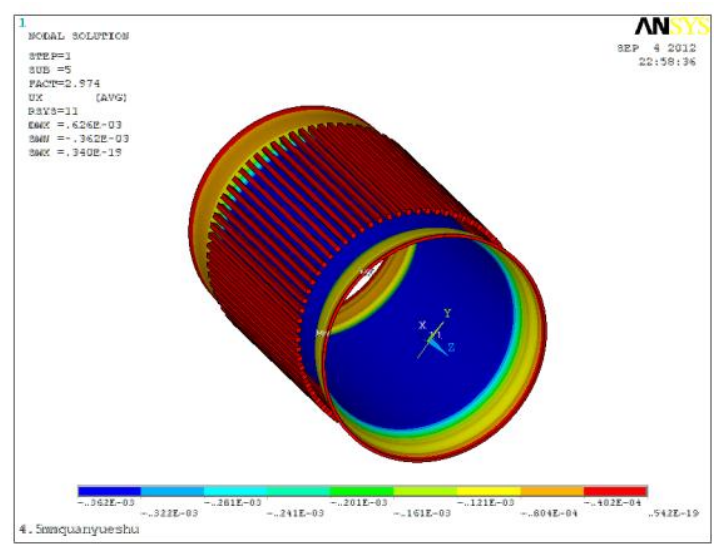

Fig. 7. The radial displacement nephogram (Core) (The maximum displacement $-0.362 \mathrm{~mm}$ )

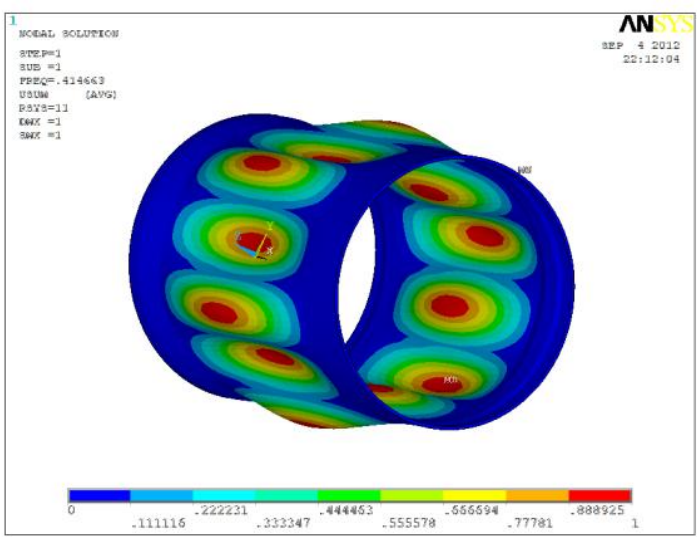

Fig. 8. First order buckling mode picture (Coreless)

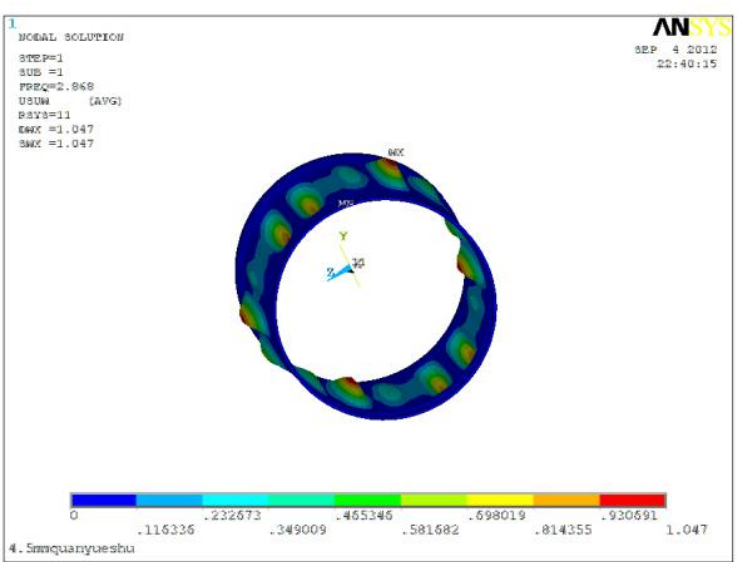

Fig. 9. First order buckling mode picture (Core)

The results also show that without stator core, the stability of the sleeve is poor (the First-order stable coefficient of only 0.4 ), but with the stator core, stability has been greatly improved to meet the application requirements (The first-order stable coefficient has reached
2.8). The epoxy resin-glass sleeve rarely causes damage because of insufficient strength; often because of inadequate stiffness instability occurs. When the load is increased to a certain value of epoxy resin-glass sleeve under external pressure, shell will suddenly lost its original shape, flat or corrugated, after the load is removed, the sleeve can not be restored, resulting in failure. Shell cross section by the original circular collapse become waveform section, shown in Fig. 10, the wave number can be equal to two, three, four or even more. When the outer stator core exists, can effectively delay further expansion sleeve suddenly deformation or prevent deformation, thus improving the stability of the sleeve, to meet the requirements of use. The statistical simulation results as shown in Table 1.

Table 1. The simulation results of stress, displacement and stability

\begin{tabular}{|c|c|c|c|}
\hline $\begin{array}{c}\text { stator } \\
\text { core }\end{array}$ & $\begin{array}{c}\text { Stress } \\
(\mathrm{MPa})\end{array}$ & $\begin{array}{c}\text { Displacement } \\
(\mathrm{mm})\end{array}$ & $\begin{array}{c}\text { Stability } \\
\text { coefficient }\end{array}$ \\
\hline No & 29.8 & -0.375 & 0.4 \\
\hline yes & 29.3 & -0.362 & 2.8 \\
\hline
\end{tabular}
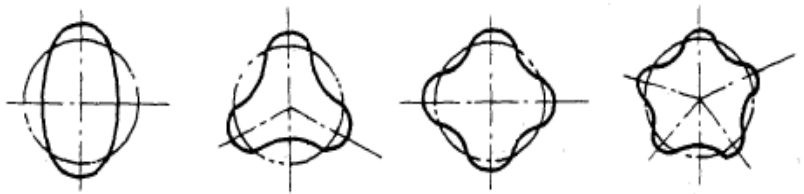

Fig. 10. Cross-sectional waveform

\section{Tests}

The sleeve of product development is installed on the motor stator (i.e. core exists) were the air tightness tested under various loading conditions, shown in Fig. 11, the tightness test load was $0.04 \mathrm{MPa}, 0.10 \mathrm{MPa}$ and $0.16 \mathrm{MPa}$. Firstly, the sleeve in the test case of the applied three kinds of load, the air tightness good, no instability, the result is consistent with the simulation calculation. At the same time, we test the change amount of the inner diameter displacement, as shown in Table 2, the sleeve radial displacement fewer than three kinds of load. The radial displacement is the average value of eight measured data is distributed in the same section of the sleeve.

In Table 2, the pressure value is the relative value of the atmospheric pressure. Radial displacement means when the load and without load the relative value of the diameter. The displacement test results show that, when the sleeve withstanding external load, there is a tendency that the displacement changes to the smaller diameter direction, and with the load growing, the displacement also increase 
gradually, the applied load caused the displacement to meet the actual requirements.

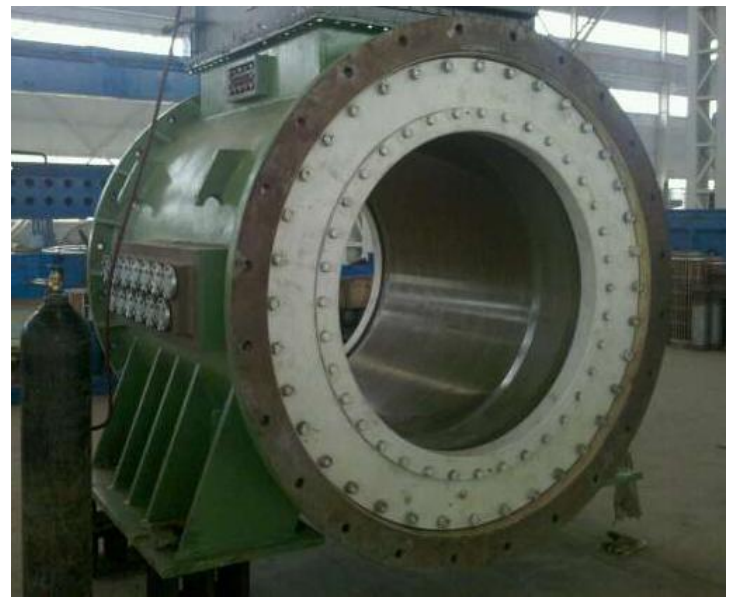

Fig. 11. Sleeve experimental test device

Table 2. the sleeve radial displacement under different load

\begin{tabular}{|c|c|c|c|}
\hline Load (MPa) & 0.04 & 0.10 & 0.16 \\
\hline Displacement (mm) & -0.14 & -0.49 & -0.59 \\
\hline
\end{tabular}

\section{Discussion and Analysis}

\subsection{The Sleeve Size Selection}

By simulation calculation, the epoxy resin-glass sleeve has high strength, under running load stress generated far less than the material allowable stress. However, its stiffness is poor, if no core strengthening effect, the sleeve cannot meet the requirements of stability. Thus, the mating dimensions of the sleeve with the stator core is very important, if the sleeve and the core excessively tight, the sleeve installation will be difficult, but the gap is too large, the core strengthening effect on the stiffness of the sleeve is reduced, this is reflected in the displacement comparison of product test and simulation. Although the simulation using the load $(0.2 \mathrm{MPa})$ than the actual test under load $(0.16 \mathrm{MPa})$ large, but its displacement produced but also smaller. One reason may be caused by error of measurement, the more important reason is that the sleeve the actual mounting size may not very closely with the stator core, there must be a certain gap, this caused the displacement of the increase. Therefore, the key is the sleeve and the stator core with a reasonable size.

In general, the gap installation may produce, in order to reduce the gap as much as possible, so that the core can greatly strengthen the sleeve stiffness, in the case of conditions, can use hot installation method. In the design of sleeve size, the size is the same as the inner diameter of the stator core, when in installation, the stator is heated to a certain temperature, thermal expansion of the core, inner diameter becomes larger, when the diameter is increased enough to install sleeve, immediately the sleeve is mounted on the stator in place, so, the stator temperature down to room temperature, the sleeve and the stator iron core gap will be as small as possible, rigidity enhancement to the sleeve will be strengthened. However, even with the heating installation method, not the sleeve diameter size design is too large, or after the installation, the core will produce stress on the sleeve, thereby affecting the mechanical strength of the sleeve.

\subsection{Choice of Coefficient of Thermal Expansion}

The actual temperature of the motor is running at between $-40^{\circ} \mathrm{C}-120^{\circ} \mathrm{C}$, Select the appropriate epoxy resinglass materials and processing technology, adjust the sleeve and the motor stator material matching coefficient of thermal expansion is not overlooked factors. Generally, the motor is running, while high temperature the greatest pressure, that is, the sleeve withstand maximum load. At this time, if the coefficient of thermal expansion of epoxy resin-glass is much larger than the stator core, at elevated temperatures, the volume expansion sleeve, so may have larger stress. Similarly, if the coefficient of thermal expansion of epoxy resin-glass is much smaller than the stator core, when the temperature rises, the gap between the sleeve and the stator core will increase, which is unfavorable stability of the sleeve. Therefore, the coefficient of thermal expansion is too large or too small are not good. By adjusting the material and processing technology, Coefficient of thermal expansion of the sleeve can be adjusted within a certain range.This paper considers the epoxy resin-glass material allowable stress is large, the use of the stator core temperature expansion coefficient equal or slightly larger is advantageous.

\subsection{Epoxy Resin-Glass Material Selection}

The sleeve is used to seal the coolant in the cavity of the stator, in addition to meet the mechanical properties of the sleeve, but also consider the problem of compatibility of sleeve material and coolant, namely the two can coexist for a long time without adverse factors. In addition, epoxy resin-glass material selection, need to be considered in all operating conditions of the motor, especially taking account of the effect of temperature on the mechanical properties of materials, epoxy resin with different sensitivity to temperature is not the same, some epoxy resin good mechanical properties at normal temperature, but above a 
certain temperature (such as $100{ }^{\circ} \mathrm{C}$ ) time, the sharp decline in performance. Therefore, to compatibility test of sleeve material and coolant, in the temperature range between $40{ }^{\circ} \mathrm{C}-120{ }^{\circ} \mathrm{C}$, temperature simulation of motor operation, observe whether the material corrosion, and mechanical properties testing, assessment materials and coolant compatibility.

\subsection{Processing Technology}

Epoxy resin-glass is a kind of composite material, great influence of different processing techniques on the performance of the product. For example, should choose to die or winding mode? If the selection of winding way, different fiber winding angle on the stiffness and strength of the sleeve is different, in the development process, need to analyze the stress state of the products, the glass fiber in the corresponding parts of the winding direction, which is very helpful for the overall performance. In addition, the reasonable selection of curing agent and curing temperature, temperature range on sleeve application is also a relationship.

\subsection{The Influence Factors of Stability}

Through calculation and test, the stability of the main influence factors of sleeve is the wall thickness and diameter, the length effect is minor. If the air gap of motor stator and rotor is fixed, it is determined the sleeve wall thickness, then the sleeve in the requirements under external pressure (such as $0.2 \mathrm{MPa}$ ) condition, the maximum diameter allowed also decided, otherwise it may lead to instability. Therefore, in the design of motor, due to changes in the sleeve wall thickness is less, if only from the stability point of view, rather than its length, and hope its diameter as small as possible.

\section{Conclusion}

Thin-walled epoxy resin-glass sleeve has high strength, good insulation, to meet the motor magnetic circuit design is very good, does not produce substantial eddy current losses. However, for large diameter thin-wall sleeve, its rigidity is poor, it can withstand greater internal pressure but relatively smaller external pressure. Therefore, in the evaporative cooling wind generator stator sleeve developed, should make full use of the motor stator core structure, rational design structure and size, increase its stiffness and improve the stability of the epoxy resin-glass sleeve, which for thin-walled the epoxy resin-glass sleeve on the successful application of wind turbines has played an important role.

Among them, there are the following three problems need special attention:

Through the design of sleeve size and reasonable installation process, control the gap between the sleeve and the stator core as small as possible;

The epoxy resin-glass materials must be compatible with the coolant;

By adjusting the epoxy resin-glass material and its processing technology, make its temperature coefficient of expansion and the stator core is consistent.

\section{Acknowledgements}

We appreciate to Institute of Electrical Engineering evaporative cooling group members for the assistance of the experiment.

\section{References}

[1] Li Shiqing, Fu Deping, Xiong Nan, "Experimental research of double evaporation cooling technology in ship propulsion motor," Advanced Technology of Electrical Engineering and Energy, 2005.

[2] Ding Shunnian, "Heating and cooling of lLarge electrical machines," Beijing: Science Press, 1992.

[3] Yu Shunzhou, Yang Jie, Tian Xindong, Guobiao, "A kind of device about stator evaporative cooling epoxy resin-glass spacer sleeve and its sealing using on wind generator," Chinese patent, 201110176584.4, 2011.

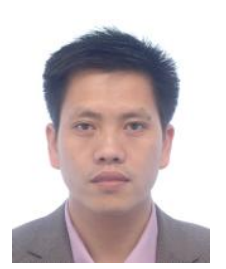

Shunzhou Yu received the B.S. degree from the Department of mechanical engineering of Tsinghua University in 2000, and M.S. degree from the electrical engineering of University of Chinese Academy of Sciences in 2003. Since 2003, he has been working at the Institute of electrical Chinese Academy of Sciences, associate professor. His current major research interests are the technology of gas-liquid phase flow and heat transfer on electrical equipment.

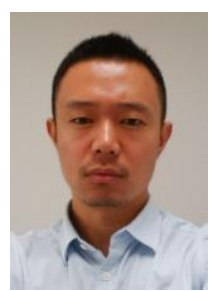

Yang Jie received B.S. degree in Electrical Engineering from Tianjin University, Tianjin, China, in 1998. He joined the Institute of Electrical Engineering, Chinese Academy of Science in 1998, engineer. His primary responsibilities include motor/ generator design and construction, thermal problems in electrical machines. 
Jiayi Yuan received B.S degree in electrical engineering from Harbin University of Science and technology in1997. He is the senior engineer of Institute of Electrical Engineering of Chinese Academy of Sciences and is currently studying for a Ph. D. degree in Harbin University of Science and technology with research interests in field of motor cooling technology and computer aided design and optimization of electric machines.

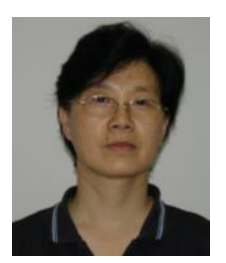

Xindong Tian has been with the Institute of electrical Chinese Academy of Sciences,serving as an senior engineer. Her main research is the evaporative cooling technology of hydro-generators and wind power generator. She attended research from the first evaporative cooling hydro generator (Yunnan Dazhai $10 \mathrm{MW}$,in 1983) to the Three Gorges 700MW evaporative cooling hydro-generator (in 2012). 